\title{
The amelioration of the suffering associated with spinal cord injury with subperception transcranial electrical stimulation
}

\author{
ID Capel $^{*, 1}$, HM Dorrell ${ }^{1}$, EP Spencer ${ }^{2}$ and MWL Davis ${ }^{3}$ \\ ${ }^{1}$ SPES Technology Ltd, Caterham, Surrey CR3 6HY, UK; ${ }^{2}$ Medical Toxicology Unit, Guy's St. Thomas' Hospital \\ Trust, Avonley Road, London SE14 5ER, UK; ${ }^{3}$ McGill University, Montreal, Quebec, Canada
}

\begin{abstract}
Study design: Double blind, partial crossover.
Objectives: To evaluate the analgesic activity of a novel cranial electrostimulus in people with spinal cord injury (SCI).

Setting: Hereward College, a residential centre that provides educational facilities for students with disabilities.

Methods: Subjects with SCI experiencing chronic pain were randomly assigned into two groups, one of which received sham and the other transcranial electrostimulation treatment (TCET) on two occasions daily for four successive days. After a 'wash-out' period of 8 weeks all subjects returned and received the identical stimulus that the treated cohort received on the first arm of the study.

Results: Pain measurements applied before and after each session indicated that the pain decreased in the treated group to $51 \%$ of that reported at the commencement of treatment; reported pain intensity did not decrease significantly in the sham treated subjects. The same (sham) subject group reported experiencing 59\% of the pain at the end of the second arm of the study (TCET) as on the first arm (sham). No significant differences were determined between the mood of all subjects estimated before and after each sham or TCET treatment session. The reported analgesic, and combined antidepressant and anxiolytic drug use in subjects receiving TCET on the second arm of the study, was $46 \%$ and $53 \%$ respectively of the average pre-study drug use. No similar decrease in the use of the drugs was noted in the same subjects after sham treatment on the first arm of the study. Salivary cortisol determinations made prior to and after each sham and treatment session implicated this corticoid in the pain-relieving mode of action of the treatment, but could not be associated with any changes in mood. Subjects receiving TCET had significantly higher urinary 3methoxy-4-hydroxy-phenylglycol (MHPG) output after the TCET treatment period than sham stimulation, implicating increased central noradrenaline (NA) metabolism in the observed effects.

Conclusion: The subjects reported less pain during, and immediately after receiving this transcranial treatment, although they were using less medication than when receiving sham treatment.
\end{abstract}

Spinal Cord (2003) 41, 109-117. doi:10.1038/sj.sc.3101401

Keywords: spinal injury; suffering; electrostimulation; subperception; transcranial

\section{Introduction}

The perception of pain is a complex process modulated by a number of factors ${ }^{1}$ whose elucidation has led to improvements in the pharmacological approach to pain amelioration. $^{2}$ The types of pain associated with spinal

*Correspondence: ID Capel, SPES Technology Ltd, Victoria House, Harestone Valley Road, Caterham, Surrey CR3 6HY, UK cord injury (SCI) however, present a major therapeutic challenge. ${ }^{3,4}$ Recent surveys ${ }^{5-7}$ of SCI subjects in various countries indicate that more than $60 \%$ of the respondents experience chronic pain/dysaesthesiae, the majority of whom describe their pain as severe. Consequently, subjects with SCI tend to be maintained on high doses and/or multiple medications from relatively young ages. ${ }^{8}$ Satisfaction, however, in 
particular with analgesic efficacy, is reportedly low ${ }^{9}$ and depression is frequently diagnosed ${ }^{6,7}$ in SCI subjects.

The suffering ${ }^{10}$ experienced by people in chronic pain should be the therapeutic target, attenuating the stressors associated with the physiological/psychological adjustments to SCI. . $^{6,9,9,11-13}$ Management of associated complaints such as sleep disturbance, ${ }^{13}$ specific type of pain $^{2-5}$ or depression ${ }^{6,7}$ increases the reliance on medication, ${ }^{8}$ some of which is contentious for long term use. ${ }^{14}$

The most familiar non-pharmaceutical alternative for pain management, transcutaneous electrical nerve stimulation (TENS), is of limited use in chronic pain ${ }^{15}$ such as that associated with SCI. A variant, spinal cord stimulation, has been used for 30 years, ${ }^{16}$ but there remains a lack of consistent proof of efficacy and cost effectiveness, and some studies ${ }^{17,18}$ report the development of tolerance with resultant loss of analgesic efficacy.

For some time non-invasive transcranial electrostimulation treatment (TCET) has been administered to ameliorate the abstinence symptoms associated with withdrawal from addictive substances ${ }^{19,20}$ and management of depression. ${ }^{21}$ There are few rigorous clinical studies to determine its value in pain management, however, although the antinociceptive action of a subperception species of TCET was demonstrated in various experimental antinociceptive models ${ }^{22,23}$ and extracellular recording techniques indicated that these transcranial stimuli modify noxious evoked responses in pain-processing regions of the brain of rats. ${ }^{24,25}$ This subperception stimulus is suited to clinical studies involving a double-blind crossover design. Furthermore, the reported anxiolytic ${ }^{19,20}$ mood-enhancing effects $^{21}$ attributed to TCET could offer additional benefits to SCI subjects, including lowering the management drug burden. Hence, total drug usage before, during and after sham or TCET was monitored as an additional indication of the efficacy of treatment (on the assumption subjects perceiving pain relief would use less medication).

To distinguish any hypoalgesic action of the TCET from reported $^{21}$ mood-enhancing effects, validated assessments often used to estimate depression and $\operatorname{mood} /$ anxiety $^{26,27}$ were administered before and after sham or TCET.

The turnover of some neurochemicals/hormones often assayed in studies evaluating mood, depression and antidepressive treatments ${ }^{28,29}$ were also monitored. Experimental studies have implicated increased central noradrenaline (NA) turnover and the hypothalamo pituitary adrenal (HPA) axis in the antinociceptive action of this TCET. ${ }^{30}$ Thus plasma adrenocortico trophic hormone (ACTH); salivary, plasma, and urinary cortisol were assayed before and after transcranial or sham treatment as objective indicators of the activity of the HPA axis in the treatment. ${ }^{28}$ The plasma concentration and total urinary output during the sham and treatment period of the principal metabolite of NA, 3-methoxy-4-hydroxy-phenylglycol (MHPG) was assayed as an indication of whether central NA turnover was implicated in any observed effects of TCET. ${ }^{29}$

The present study was conducted to evaluate the therapeutic efficacy of this novel transcranial stimulus to ameliorate suffering. The additional assessments to determine the effect of TCET on mood/depression were conducted to indicate whether any observed analgesic action was secondary to a mood enhancing action or, as might be anticipated from the antinociceptive action in experimental animals, ${ }^{22-24}$ was an independent analgesic effect.

\section{Materials and methods}

\section{Subjects}

Paraplegic and tetraplegic individuals totalling 30 volunteers in all were located amongst their membership by the Spinal Injuries Association, London, UK. These subjects agreed to attend a residential centre (Hereward College, Coventry, UK which provides educational facilities for students with disabilities) on two separate occasions of 5 days, where they would receive $2 \times$ TCET or $2 \times$ sham treatments daily, one between $10.00-$ $12.00 \mathrm{~h}$ and the other $14.00-16.00 \mathrm{~h}$ on 4 successive days. All participants agreed to record all medication used during the 3 days preceding, the 4-day test period and 7 days succeeding each arm of the study. All volunteers included also agreed to reduce and (if possible) cease using analgesic, anxiolytic or antidepressive medication $24 \mathrm{~h}$ prior to commencing TCET or sham treatment and to refrain from consuming alcohol during each sham or treatment period.

The participants comprised nine subjects with cervical, 16 with thoracic and the remainder with lumbar lesions. All subjects presented with a history of chronic pain described as neuropathic, nerve root entrapment, visceral, musculoskeletal and of undiagnosed origins. Smokers and subjects with other conditions or using medications contraindicating the (then) known exclusion criteria did not participate. Three of the 30 subjects included were withdrawn from the study after commencement, one of whom developed an upper respiratory infection, and two others were withdrawn from the study because their medication (either $\mathrm{H} 2$ antagonist anti-ulcer or steroidal inhalant) were interacting with the TCET treatment. All subjects were free to withdraw from the study, none voluntarily withdrew.

\section{Study design}

The study was designed to occur in two arms with an 8 -week wash-out period between. Subjects would be randomly assigned into two groups according to their choice of treatment device. Thirty treatment devices were provided, 15 delivered TCET and 15 others (sham) appeared to be delivering treatment because the 
indicators functioned in the same manner as the TCET devices. The devices were numbered for identification, but neither the administrators nor the recipients of the treatment could distinguish between the devices. On the first arm of the test, before the first session, the subjects were invited to choose a device. The chosen device remained with that subject throughout the study. It was anticipated that at the end of the first arm of the study all the devices would be returned to the engineers for their functions to be reversed so that the TCET would become sham devices and vice versa. After an independent statistical analysis of the results of the first arm of the study, it was decided to reverse only the sham treatment devices rather than withhold effective treatment from subjects who were being requested to cease using their existing medication. Therefore, on the second arm of the study, all the subjects received TCET treatment.

\section{Equipment}

TCET or sham treatment was administered by purpose-made portable signal generators constructed by Carex, Hemel Hempstead, UK. All devices had LEDs to indicate when treatment was, or was apparently (in the case of sham treatment) being delivered and when it was completed. An alarm indicated if the subject became inadvertently disconnected. TCET was effected via customised earpieces attached to each ear lobe. The earpieces contained pin electrodes that pierce only the outermost layers of the skin, facilitating a consistent low impedance. 'Smart' circuitry within the device compensated for alterations in conduction and/or capacitance during treatment, so that every TCET-treated individual received an identical treatment paradigm. There were no user controls/adjustments on the equipment. The treatment cycle was initiated by the insertion of the lead connected to the earpieces into the device, the cranium of the subject completed the circuit, thus effecting the passage of an exact standardised current. All subjects were monitored while receiving TCET or sham treatment. Any subject who became disconnected was immediately reconnected. The sham and TCET treatment sessions lasted $53 \mathrm{~min}$. Each TCET-treated subject received interrupted trains of charge-balanced impulses of positive amplitude $12 \mu \mathrm{A}$ and positive duration $2.0 \mathrm{msec}$ at a duty cycle of $10 \mathrm{cps}$. Both TCET and sham devices were tested on an oscilloscope before and after all treatment sessions.

\section{Assessment procedures}

The characteristics and intensity of the pain was assessed using the self-reported Short Form McGill Pain Questionnaire (SF-MPQ) ${ }^{31}$ which provides a Pain Rating Index (PRI) based on the total score of adjective words selected by the subject; a Present Pain Index (PPI) based on a $0-5$ intensity scale and Visual Analogue Scale (VAS) based on the level of pain noted on a $10 \mathrm{~cm}$ scale line. Anxiety, depression and neuroticism are confounding characteristics that influence pain perception and these were also determined by self-reporting. Anxiety was assessed using the Trait form X2 (comprising 20 statements scored on a 1-4 intensity scale) of the Speilberger State and Trait Anxiety Inventory (STAI). ${ }^{27}$ Only the Trait part of the STAI was used as it is relatively impervious to the conditions under which it is administered, whereas the State part of the STAI demands exact parameters not met in the study (eg quiet room, same time every day, etc.). The short form Beck Depression Inventory $(\mathrm{BDI})^{26}$ based on total score of 13 statements ranging in severity from $0-3$ was used to assess affective symptoms indicative of depression, and the Eysenck Personality Inventory (EPQ) ${ }^{32}$ to screen for neuroticism. Subjects completed the SF-MPQ questionnaire before commencement and on finishing each treatment session on each of the 8 days of the study. The STAI, BDI and EPQ questionnaires were completed before commencement on the first day and at the end of each of the 4-day periods on each arm of the study, where necessary assistance was provided by the nursing staff. The questionnaires were collated and forwarded to professional statisticians, Priority Search, Sheffield, UK, who analyzed the data after the first and second arm of the study, unaware as to which group received TCET and which received sham treatment.

Differences in medication use throughout the study were compared where possible. Some subjects had automatic delivery systems implanted, others reported not receiving any medication that would impact on suffering, specifically, analgesics, antidepressants and anxiolytics, at the time of each arm of the study.

\section{Assessment of medication use}

The type of medication used was categorised by purpose as analgesic (including anti-inflammatory), anxiolytic including hypnotics and muscle relaxants, antidepressives, antispasmodics, antibiotics, cardiovascular agents, diuretics, laxatives, bulking agents, antiulcer therapy and HRT. Some subjects reported that their antidepressant medication, in particular tricyclic antidepressants (TCADs) were prescribed as analgesic adjuvants. Where, however, the dosage was prescribed at the level indicated for treatment of depression, the medication was categorised as antidepressive. The use of analgesics and combined anxiolytic/antidepressants before, during, and after receiving TCET or sham treatment, was compared in the same subjects.

\section{Sample collection for assays}

Salivary samples $(4 \mathrm{ml})$ were collected into tubes before commencement and immediately after completion of all TCET or sham treatments. These samples were immediately frozen in solid $\mathrm{CO}_{2}$ for transportation and storage in liquid $\mathrm{N}_{2}$ prior to assay. 
Most of the participants had catheters and their urine collected into leg-bags. Subjects who were able to provide samples voided their bladder completely before and immediately after all TCET or sham treatments. Leg-bags were emptied completely prior to and immediately upon completion of TCET or sham treatment. The total volume of each sample was recorded and two separate aliquots of each sample $(20 \mathrm{ml})$ frozen immediately in solid $\mathrm{CO}_{2}$ prior to transportation and storage in liquid $\mathrm{N}_{2}$.

Blood $(10 \mathrm{ml})$ was collected by venepuncture from the antecubital vein before the first TCET or sham treatment prior to commencement of the first, and after completion of the last session, on each procedure day. No haemolysed samples were accepted. Some subjects, after receiving one of the treatment paradigms, were difficult to sample even when using indwelling catheters. This was associated with an observed general decrease in blood pressure in one of the treatment groups. Whole blood, collected and placed into EDTA-coated tubes, was separated by centrifugation $\left(1500 \mathrm{~g}\right.$ av for $20 \mathrm{~min}$ at $\left.2^{\circ} \mathrm{C}\right)$. The resultant plasma was drawn off and aliquoted into two portions $(2 \mathrm{ml})$ and frozen immediately in solid $\mathrm{CO}_{2}$ for transportation and storage in liquid $\mathrm{N}_{2}$.

\section{Assays}

Operatives analysing plasma, saliva and urine samples were unaware of the regimen of treatment (TCET or sham) the subjects had received. Urinary, plasma and salivary cortisol concentration was determined by radioimmuno assay (RIA) using the antisera supplied in the Amerlex RIA kit provided by Amersham International plc (Amersham, UK), essentially by the method of Walker et $a l^{33}$ except that the salivary steroid was not extracted prior to assay, and the saliva was centrifuged to remove particulate matter. Plasma ACTH concentration was determined by RIA using the antisera and kit supplied by Incstar Corporation, Minnesota, USA. Plasma and urinary MHPG concentrations were determined by reversed-phase high performance liquid chromatography (HPLC) with electrochemical detection by a method based on that described by Taylor et $a l^{34}$ after the conjugated MHPG had been completely hydrolysed essentially as described by Warnhoff. ${ }^{35}$

\section{Statistical analysis}

The average of reported pain values for each individual in the TCET or sham treated group following each treatment session was determined after each arm of the study. The statistical significance of changes against time between the two groups for each of the 16 average values on each arm of the study was determined by Spearman Rank Correlation, and comparison between the pain intensity in the two groups at commencement of the first arm was determined by the Fisher Exact Probability Test. The statistical significance of differ- ences between medication use amongst the same subjects who received sham treatment on the first arm of the study and TCET on the second arm were determined by a two-tailed Wilcoxon-Mann-Whitney test. The statistical significance of differences in the individual values of the concentration of cortisol, ACTH and MHPG in plasma after sham or TCET treatment, was determined by one-way ANOVA followed by Tukey's multiple comparison test. In the case of salivary cortisol concentration, each individual post-treatment value was expressed as a percentage of the corresponding pre-treatment value to lessen the influence of individual variation between the subjects. The statistical significance of percentage changes in the concentration of salivary cortisol following TCET or sham treatment over the corresponding pre-treatment value was determined by one-way ANOVA followed by Tukey's multiple comparison test. The statistical significance of differences in the individual values of total excretion of MHPG and cortisol in the urine after TCET or sham treatment was compared by one-way ANOVA followed by Tukey's multiple comparison test.

\section{Results}

\section{Acceptance of treatment}

Vital signs were taken of all subjects before and after completion of each TCET or sham session. No adverse reactions to either regimen were reported. Some subjects, specifically those receiving TCET, did report a feeling of relaxation that coincided with lowered blood pressure. Venepuncture was therefore not always possible post-TCET treatment.

\section{Personality and mood variables}

The scores of both groups on the four variables of the EPQ were compared and both groups did not differ significantly from the reported population norm in extraversion, neurosis, psychosis and truthfulness. There were no significant correlations between the overall pain improvement scores of individuals and each of the EPQ variables indicating that the hypoalgesic effects of the TCET were independent of personality variables. No significant change associated with TCET against sham treatment was determined by comparison of the bipolar profile of mood states for anxiety, hostility, depression, lethargy, confusion and unsureness. A positive effect of the study was detected irrespective of the treatment (TCET or sham) implying that mood change was a non-specific event associated with the study but unrelated to treatment received.

\section{Reported pain ratings}

Comparison between the initial severity of reported pain on the first arm of the study between the subjects 
before receiving TCET or sham treatment revealed no significant difference by the Fisher Exact Probability Test. The same comparison at the end of the first arm of the study indicated that the reported intensity of the pain of the TCET treated subjects was significantly lower $(P=0.0016)$ than the different subjects who had received sham treatment. Subjects receiving sham treatment had no consistent change in reported pain over the 16 observations of the first arm of the study $\left(r_{s}=1.17, P=0.50\right)$. Subjects receiving TCET treatment had consistent improvement $\left(r_{s}=0.86, P<0.001\right)$ in their pain score on the SF-MPQ compared with the different subjects receiving sham treatment over the 16 observations on the first arm of the study (Figure 1). The same subjects receiving TCET on the second arm of the study, who had received sham treatment on the first arm of the study, showed significant $\left(r_{s}=0.68\right.$, $P<0.005)$ improvement over the 16 measurements when they received TCET (second arm) compared with when they received sham (first arm) of the study (Figure 2).

\section{Reported drug usage}

At commencement there was no significant difference in the average number of medications used by the same subjects on the first arm (sham) and the second arm (TCET treatment), averaging 2.92 and 2.88 drug categories per person, respectively. Comparing the analgesic and combined anxiolytic and antidepressant drug usage on each arm of the study, however, the same subjects receiving TCET reported using significantly $(P<0.05)$ less $(46 \%$ of the average pretreatment level) analgesic medication and significantly

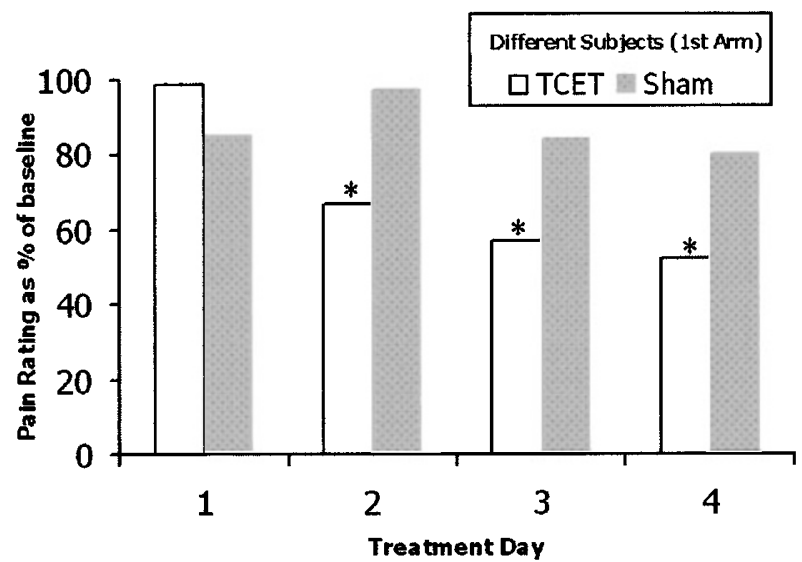

Figure 1 The bars represent the average daily pain rating (mean of four measurements per day-pre and post AM, pre and post PM session) for groups of different subjects receiving either Sham $(n=13)$ or TCET $(n=14)$ treatment (1st arm of study), expressed as a per cent of the baseline values. The baseline value is the summed and averaged pain(s) experienced by the subjects prior to commencement of the study. ${ }^{*}$ Significantly different $(P<0.05)$ from Sham value
$(P<0.05)$ less $(53 \%)$ of the average pre-treatment level of combined antidepressant and anxiolytic medication during the four treatment days (second arm) of the study. No significant decrease in use of any of these medications occurred in the first arm of the study when they received sham treatment. Furthermore, the reported use of these medications was significantly $(P<0.05)$ reduced during the week succeeding TCET treatment but not during the week succeeding sham treatment (Figure 3). There were too few subjects reporting no drug usage at commence-

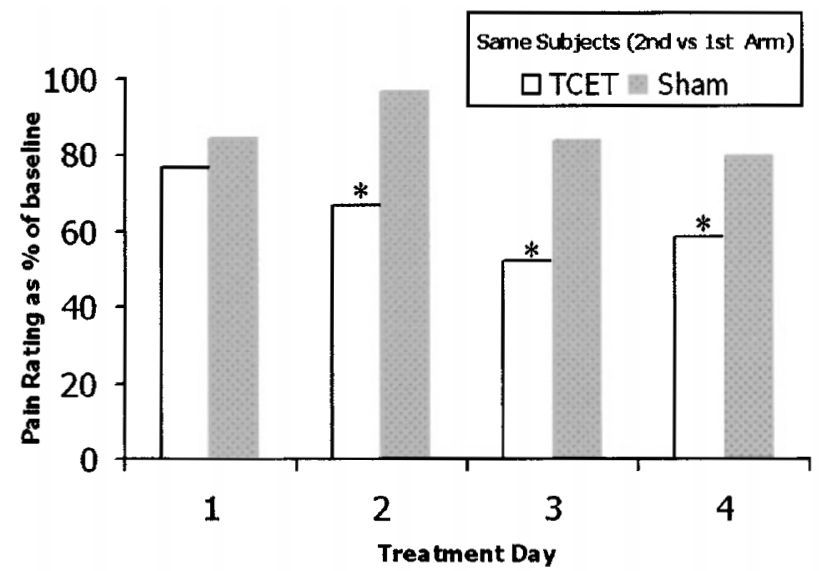

Figure 2 The bars represent the average daily pain rating (mean of four measurements per day-pre and post AM, pre and post PM session) for groups of subjects receiving either Sham (first arm of study, $n=13$ ) or TCET (second arm of study, $n=13$ ) treatment, expressed as a per cent of the baseline values. The baseline value is the summed and averaged pain(s) experienced by the subjects prior to commencement of the study. *Significantly different $(P<0.05)$ from Sham value

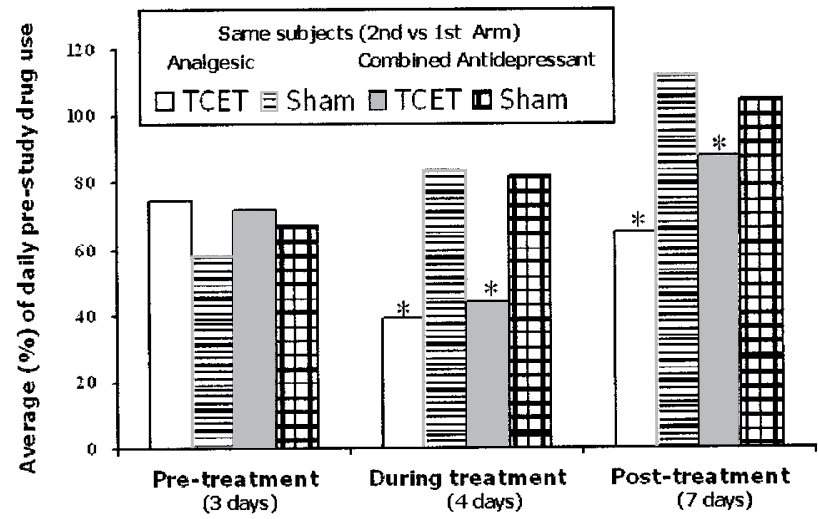

Figure 3 The bars represent the average analgesic (TCET $n=8$, Sham $n=6$ ) and combined anxiolytic/antidepressant drug use (TCET $n=10$, Sham $n=11$ ) per day for groups of the same subjects receiving either Sham (first arm study) or TCET (second arm study) over 4 days; for 3 days preceding commencement of the study and for the 7 days following the study. *Significantly different $(P<0.05)$ from Sham value 
ment of the study to determine whether there were any differences in the effects of the TCET treatment in drug-free compared with medication-using participants.

\section{Biochemical assays}

Plasma assays There were no significant differences between plasma cortisol concentration before and after either treatment amongst different subjects, TCET versus sham first arm of the study, or comparing the same group of subjects, first versus second arm of the study. There was a significant diurnal $(F[7360]=13.06$, $P<0.05)$ difference between the morning and afternoon values (Table 1).

There were no significant differences between the actual ACTH concentrations comparing percentages (when the post-treatment was expressed as a percentage of the pre-treatment concentration) between subjects receiving sham or TCET treatment (Table 1).

A high individual variation in plasma MHPG concentration before and after TCET or sham treatment was observed. No statistical differences in plasma MHPG were observed between TCET and sham treatment in different subjects on the first arm, or comparing the same subjects on the first (sham) versus the second (TCET) arm of the study (Table 1).

Salivary assays Large individual variations between salivary cortisol concentrations were observed. When the post-treatment concentration of steroid was expressed as a percentage of the pre-treatment value, marked differences between the TCET and sham treated groups were noted. The average salivary concentration (post/pre value as per cent) of cortisol was significantly $(F[7424]=13.38, P<0.05)$ lower in the different subject groups which received TCET compared with sham treatment (first arm of the study). The average salivary concentration of cortisol was significantly $(F[3204]=10.57, P<0.05)$ lower than in the same subject groups which received TCET (on the second arm) than when they received sham treatment (on the first arm of the study). There was no significant difference between the decreased salivary cortisol concentration when comparing the same subject groups receiving TCET on the first and second arm of the study. The statistically significant differences in salivary cortisol concentration following TCET and sham treatment were apparent whether the morning and afternoon treatment sessions were compared separately or together (Table 1).

Urinary assays Individual urinary cortisol excretion was extremely variable after morning or afternoon sham or TCET treatment. No significant differences between the same subjects receiving sham and TCET or in different subjects receiving sham or TCET was observed.

Considering the total MHPG excreted during sham or TCET treatment, and comparing the morning and afternoon treatment sessions separately, or together, all subjects receiving TCET excreted significantly $(F$ $[7424]=17.88, P<0.05)$ more MHPG in their urine. Urinary MHPG excretion was significantly $(F$

Table 1 Plasma ACTH, cortisol and MHPG; urinary cortisol and MHPG; salivary cortisol in subjects with SCI receiving either TCET or sham treatment

\begin{tabular}{|c|c|c|c|c|c|}
\hline & & First arm & $t u d y$ & Second ar & m study \\
\hline & & Sham & TCET & TCET (sham first) & TCET (TCET first) \\
\hline Plasma & & & & & \\
\hline ACTH (pg/ml) & $\mathrm{AM}$ & $37.0 \pm 6.6(44)$ & $39.0 \pm 11.6(48)$ & $37.1 \pm 8.2(44)$ & $41.1 \pm 14.0$ \\
\hline & PM & $38.9 \pm 8.9(44)$ & $39.6 \pm 9.9(48)$ & $40.9 \pm 1.5(44)$ & $42.8 \pm 15.9(48)$ \\
\hline Cortisol (ng/ml) & AM & $206 \pm 82(44)$ & $180 \pm 51(48)$ & $195 \pm 73(44)$ & $191 \pm 35(48)$ \\
\hline & PM & $155 \pm 76(44)$ & $132 \pm 51(48)$ & $140 \pm 53(44)$ & $124 \pm 41(48)$ \\
\hline MHPG (ng/ml) & AM & $9.5 \pm 3.3(44)$ & $11.7 \pm 4.1(48)$ & $9.6 \pm 4.7(44)$ & $11.7 \pm 2.9(48)$ \\
\hline & PM & $9.9 \pm 4.8(44)$ & $10.4 \pm 3.7(48)$ & $10.2 \pm 9.0(44)$ & $10.5 \pm 2.5(48)$ \\
\hline Urine & & & & & \\
\hline Cortisol (total $\mu \mathrm{g} / \mathrm{h}$ ) & $\mathrm{AM}$ & $11.9 \pm 12.0$ & $10.4 \pm 9.5(56)$ & $5.5 \pm 3.7(52)$ & $8.1 \pm 7.2(56)$ \\
\hline & PM & $12.9 \pm 17.9(52)$ & $6.9 \pm 4.6(56)$ & $7.3 \pm 6.9(52)$ & $6.4 \pm 4.8(56)$ \\
\hline MHPG (total ng/h) & $\mathrm{AM}$ & $732 \pm 605(52)$ & $1655 \pm 970(56)^{*}$ & $2103 \pm 1722(52)^{*}$ & $1788 \pm 855(56)^{*}$ \\
\hline & $\mathrm{PM}$ & $655 \pm 654(52)$ & $1557 \pm 830(56)^{*}$ & $1164 \pm 766(52)^{*}$ & $2061 \pm 866(56)^{*}$ \\
\hline Saliva & & & & & \\
\hline Cortisol (total \%post/pre) & $\mathrm{AM}$ & $79.8 \pm 36.7(52)$ & $55.1 \pm 18.2(56)^{*}$ & $57.4 \pm 19.9(52)^{*}$ & $49.1 \pm 16.9(56)^{*}$ \\
\hline & PM & $84.4 \pm 30.9(52)$ & $66.8 \pm 22.7(56)^{*}$ & $62.9 \pm 24.9(52)^{*}$ & $57.7 \pm 20.6(56)^{*}$ \\
\hline
\end{tabular}

All values represent the mean \pm SD of four consecutive days observations pooled (total number of observations in parenthesis). Salivary cortisol $(\mathrm{ng} / \mathrm{ml})$ is expressed as per cent of post/pre treatment values. The observations are for $11-13$ individuals in the sham group and 12-14 individuals in the TCET group for the first arm of the study. All individuals received TCET in the second arm of the study. Statistical comparisons between the four groups of individuals were made by one-way ANOVA followed by Tukey's multiple range test, differences were considered significant when $P<0.05$. *Significantly different from sham treated value. 
$[3212]=24.56, P<0.05)$ greater in subjects receiving TCET than sham treatment in the first arm of the study, and significantly higher $(F \quad[3204]=21.14$, $P<0.05)$ when TCET was received on the second arm by subjects who had received sham in the first arm of the study (Table 1).

\section{Discussion}

The term 'suffering' has been described as a nebulous concept ${ }^{10}$ but such a 'holistic' expression encompasses both the chronic pain ${ }^{4-9,13}$ and stressors, ${ }^{11,12}$ associated with SCI. Depression, for example, occurs in up to $40 \%$ of SCI subjects, and a recent review indicates post-traumatic stress disorder could be prevalent in $50 \%$ of the people with SCI. ${ }^{36}$ Such psychobiological variables influence the neurochemical response to medication $^{37}$ and these associated stressors could contribute to the intractable nature of the pain associated with SCI.

The medication use by SCI subjects is typically high $^{8}$ and their satisfaction with their treatment reportedly low. ${ }^{9}$ Tolerance and/or dependence may develop to some medication used in the control of pain $^{14}$ and anxiety/depression, ${ }^{38}$ and since many SCI subjects are relatively young, long-term management of SCI needs new developments.

Non-drug treatments such as TENS and spinal cord stimulation are administered to ameliorate pain. ${ }^{15-17}$ In view of the intricate involvement of psychological variables described in pain, transcranial treatment could provide ${ }^{19-22}$ a more comprehensive therapy for the management of SCI. The TCET species employed in the present studies was identified using standard pharmaceutical testing models for the development of new analgesic agents. The administration of the stimulus under test conditions necessitated restraint procedures. ${ }^{22,23,30}$ It was determined that the most effective antinociceptive signal did not elicit its response through mechanisms collectively described as Stress Induced Analgesia (SIA), rather, this TCET species ameliorated stress, and its antinociceptive action was temporally and neurochemically distinct from SIA $^{23,30}$ being more similar to stimulation provoked analgesia (SPA). ${ }^{24,25}$

In the present study TCET treatment ameliorated SCI-associated pain reported in the recipients compared with sham treatment, but the psychological instruments employed indicate that the hypoalgesic effect was independent of changes in mood, whereas the biochemical indices indicated that the TCET has some similar neurochemical actions in the SCI subjects as in the experimental models. ${ }^{30}$

The pain reported by the subjects included in the study was categorised into five sub-divisions. With the relatively small sample size and the presence of more than one pain sub-type in most subjects it was not possible statistically to support greater efficacy for TCET in any particular pain sub-type compared with another. It was interesting to note that all the participants experiencing visceral pain/gut cramps voluntarily commented on benefits associated with TCET (but not sham) treatment.

The relatively small number of subjects, large individual variations, morning and afternoon differences, and the difficulties in sampling, diminished the value of the blood estimations, hence with the exception of total plasma cortisol, even diurnal differences were not clearly apparent. However, salivary, ie free cortisol, was determined as a more sensitive index of changes in the HPA function than measuring total plasma cortisol. ${ }^{28}$ Whereas lowered salivary cortisol has been associated with depression, ${ }^{28}$ decreases in salivary cortisol have been associated with mood enhancement in certain groups of patients who had elevated salivary cortisol at the outset. ${ }^{39}$ In the present study elevated cortisol would be anticipated as a consequence of chronic pain, the decreased concentration following TCET reflecting an ameliorating effect on the pain (stress) which provoked the raised cortisol excretion.

Urinary MHPG is often assayed ${ }^{29,40}$ as an index of central NA activity. The comparatively higher urinary MHPG output following TCET as opposed to sham treatment indicates increased turnover of NA and increased activity of this biogenic amine. Although the reports are not unequivocal, low levels of MHPG are usually associated with depressive spectrum disorder, ${ }^{41}$ while raised nocturnal urinary MHPG has been observed in combat-related PTSD. ${ }^{42}$ In the experimental studies ${ }^{30}$ increased activity of NA (and in particular increased MHPG concentration) in different brain regions and activation of the feedback control exerted via the HPA axis is obligatory for the antinociceptive action of TCET.

It was concluded that the TCET was probably exerting a similar anti-stress action in the SCI subjects as in experimental studies, ${ }^{23,30}$ but differences in mood enhancement comparing TCET and sham treatments were obscured by the psychosocial variables introduced by the study. ${ }^{43}$ These were essentially positive reinforcements, such as environmental novelty, the high level of care and attention provided at the venue of the study and inter-individual support and social interactions with fellow SCI subjects, together with the active connection to a sham device which appeared to be functioning properly.

Another factor which could influence mood was the voluntary self-reported withdrawal from analgesic, antidepressive and anxiolytic therapy during the study. Urinary MHPG excretion does alter in unipolar and bipolar depressed subjects receiving TCADs. ${ }^{44}$ Therefore, the observed differences in biochemical indices could have been a direct result of decreased drug use. The levels of those parameters, which showed consistently statistically significant changes, namely salivary cortisol and urinary MHPG, were fairly constant pre-TCET treatment however, and the decrease in cortisol and increase in MHPG was seen immediately after TCET, but not sham treatment. 
The same subjects who had received TCET had decreased requirements for some medication during treatment and on the week succeeding TCET, than during or after the arm of the study when they received sham treatment. This present TCET species has been demonstrated to reduce the abstinence effects associated with withdrawal from morphine in experimental models of addictive behaviour. ${ }^{45,46}$ It would therefore be anticipated that TCET could ameliorate the distressing symptoms associated with abrupt withdrawal from medication that the SCI subjects might have encountered. However, on a short-term (four successive days study) it is unsafe to conclude that this decreased requirement would be maintained. Drug usage, although higher than during the treatment period, was still significantly less than their pre-study average in the week succeeding TCET. This increasing medication use in the subjects in the week after cessation of TCET treatment could have been exacerbated by negative affectivity ${ }^{47}$ upon completion of the study, which could have increased the various chronic pain experiences returning in the absence of treatment. Alternatively, the participants could have voluntarily resumed their pre-study treatment regimens in the absence of further access to the TCET. Larger scale and longer-term studies are required to determine whether transcranial treatment could lower the longterm medication necessary for the management of the problems associated with SCI.

This study does suggest, however, that TCET treatment presently indicated for the amelioration of stress-related conditions and withdrawal from addictive substances could be useful in the management of pain and the overall suffering associated with SCI, and that larger scale studies designed to evaluate specifically beneficial action on depression or PTSD in SCI should be conducted.

\section{Acknowledgements}

The authors are indebted to the Laing Foundation, Thera Trust and Smith's Charity for financial assistance. The authors are also grateful to the excellent staff and for the facilities provided by Hereward College and to the Duracell Battery Company for assistance with equipment. Most of all, the authors gratefully acknowledge the contribution of the Spinal Injuries Association of the $\mathrm{UK}$, and, in particular, the input of Mr Stephen Bradshaw, without whose help, advice and encouragement, this study would not have occurred.

\section{References}

1 Loeser JD, Melzack R. Pain: an overview. The Lancet 2001; 357: $1607-1609$.

2 Besson JM. The neurobiology of pain. The Lancet 1999; 353: $1610-1615$.

3 Eide PK. Pathophysiological mechanisms of central neuropathic pain after spinal cord injury. Spinal Cord 1998; 36: $601-612$.
4 Siddal PJ, Loeser JD. Pain following spinal cord injury. Spinal Cord 2001; 39: 63-73.

5 Finnerup NB et al. Pain and dysethesia in patients with spinal cord injury: A postal survey. Spinal Cord 2001; 39: $256-262$

6 Ravenscroft A, Ahmed YS, Burnside IG. Chronic pain after SCI. A patient survey. Spinal Cord 2000; 38: 611 614.

7 Stormer S et al. Chronic pain/dysaesthesiae in spinal cord injury patients: results of a multi-centre study. Spinal Cord 1997; 35: 446-455.

8 Hope ME, Kallis SG. Medication usage in a spinal cord injured population. Spinal Cord 1998; 36: 161-165.

9 Murphy D, Reid DB. Pain treatment satisfaction in spinal cord injury. Spinal Cord 2001; 39: 44-46.

10 Chapman CR, Gavrin J. Suffering: the contributions of persistent pain. The Lancet 1999; 353: 2233-2237.

11 Gerhart KA et al. Correlates of stress in long-term spinal cord injury. Spinal Cord 1999; 37: 183-190.

12 North NT. The psychological effects of spinal cord injury a review. Spinal Cord 1999; 37: 671-679.

13 Biering-Sørensen F, Biering-Sørensen M. Sleep disturbances in the spinal cord injured: an epidemiological questionnaire investigation, including a normal population. Spinal Cord 2001; 39: $505-513$.

$14 \mathrm{McQuay} \mathrm{H}$. Opioids in pain management. The Lancet 1999; 353: 2229-2232.

15 Marchand S et al. Is TENS purely a placebo effect? - a controlled study on chronic low back pain. Pain 1993; 54: $99-106$.

16 Vaarwerk IAT, Staal MJ. Spinal cord stimulation in chronic pain syndromes. Spinal Cord 1998; 36: 671 - 682 .

17 Kupers RC et al. Spinal cord stimulation in Belgium - a nationwide survey on the incidence, indications and therapeutic efficacy by the health insurer. Pain 1994; 56: $211-216$.

18 Cioni B et al. Spinal cord stimulation in the treatment of paraplegic pain. J Neurosurg 1995; 82: 35-39.

19 Braverman E et al. Modification of P300 amplitude and other electrophysiological parameters of drug abuse by cranial electrical stimulation. Current Therapeutic Res 1990; 48: $586-596$.

20 Krupitsky EM et al. The administration of transcranial electric treatment for affective disturbances therapy in alcoholic patients. Drug and Alcohol Dependence 1991; 27: $1-6$.

21 Philip P. Efficiency of transcranial electrostimulation on anxiety and insomnia symptoms during a washout period in depressed patients: a double-blind study. Biolog Psych 1991; 29: $451-456$.

22 Wilson $\mathrm{O}$ et al. The influence of electrical parameters on the analgesia produced by low current transcranial electrostimulation of rats. J Anes Analg 1989; 8: 673-681.

23 Capel ID, Dorrell HM, Spencer EP. The application of sub-perception electrical stimuli elicits a temporally distinct response from restraint stress: I. antinociceptive characteristics. J Bioelectricity 1990; 9: 167-176.

24 Qiao JT, Skolnick MH, Dafny N. Dorsal raphe and external electrical stimulation modulate noxious input to single neurons in nucleus parafascicularis thalami. Brain Res Bull 1988; 21: 671-675.

25 Dong WQ et al. Hypothalamic, dorsal raphe and external electrical stimulation modulate noxious evoked responses of habenula neurons. Neurosci 1992; 48: $933-$ 940. 
26 Beck AT, Beck RW. Screening depressed patients in family practice: a rapid technique. Postgrad Med 1972; 52: $81-85$.

27 Spielberger CD, Gorsuch RL, Lushene RE. The statetrait anxiety inventory (STAI) - test manual for form $X$. Palo Alto: California Psychological Press, 1968.

28 Kirschbaum C, Hellhammer DH. Salivary cortisol in psychobiological research: an overview. Neuropsychobiol 1989; 22: $150-169$.

29 Grossman F, Potter WZ. Catecholamines in depression: a cumulative study of urinary norepinephrine and its major metabolites in unipolar and bipolar depressed patients versus healthy volunteers at the NIMH. Psychiatry Res 1999; 87: $21-27$.

30 Capel ID, Dorrell HM, Spencer EP. The application of sub-perception electrical stimuli elicits a temporally distinct response from restraint stress: II. Neurochemical considerations. J Bioelectricity 1990; 9: $177-186$.

31 Melzack R. The short-term McGill pain questionnaire. Pain 1987; 30: $191-197$.

32 Eysenk JH, Eysenk SBG. The Eysenk personality inventory. In: San Diego Educational and Industrial Testing Service. London: University of London Press, 1963.

33 Walker RF et al. Adrenal status assessed by direct RIA of cortisol in whole saliva or parotid saliva. Clin Chem 1978; 24: $1460-1463$.

34 Taylor RB et al. Assay procedures for the determination of biogenic amines and their metabolites in rat hypothalamus using ion-pairing reversed-phase high-performance liquid chromatography. J Chromatog 1983; 277: $101-114$.

35 Warnhoff M. Simultaneous determination of norepinephrine, dopamine, 5-hydroxytryptamine and their main metabolites in rat brain using high-performance liquid chromatography with electro-chemical detection: Enzymatic hydrolysis of metabolites prior to chromatography. J Chromatog 1984; 307: $271-281$.

36 Kennedy P, Duff J. Post traumatic stress disorder and spinal cord injuries. Spinal Cord 2001; 39: $1-10$.
37 Linton SJ, Skevington SM, Crombie IK et al. (eds). Psychological factors. In: Epidemiology of Pain a report of the Task Force on Epidemiology of the international Association for the Study of Pain. Seattle: IASP Press, 1999; pp 25-42.

38 Lango LP, Johnson B. Addiction: Part 1. Benzodiazepines - side effects, abuse risk and alternatives. Am Fam Physician 2000; 61: $2121-2128$

39 Cruess DG et al. Reductions in salivary cortisol are associated with mood improvement during relaxation training among HIV-seropositive men. J Behav Med 2000; 23: $107-122$.

40 Roy A. Norepinephrine and its metabolites in cerebrospinal fluid, plasma and urine. Relationship to hypothalamic-pituitary-adrenal axis function in depression. Arch Gen Psychiatry 1988; 45: 849-857.

41 Garvey MJ, Tuason VB. Do low levels of MHPG in depressive spectrum patients normalise after successful treatment? Neuropsychobiol 1996; 34: 188-191.

42 Mellman TA et al. Nocturnal/daytime urine noradrenergic measures and sleep in combat-related PTSD. Biol Psychiatry 1995; 38: $174-179$.

43 Hrobjartsson A, Gotzsche PC. Is the placebo powerless? New Engl J Med 2001; 344: 1594-1602.

44 Bowden CL et al. Changes in urinary catecholamines and their metabolites in depressed patients treated with amitriptyline or imipramine. J Psychiatr Res 1987; 21: $111-128$

45 Malin DH et al. Auricular microelectrostimulation; naloxone reversible attenuation of opiate abstinence syndrome. Biol Psych 1988; 4: 886-890.

46 Doughty PM et al. Trans-cranial electrical stimulation attenuates abrupt morphine withdrawal in rats assayed by remote computerised quantification of multiple motor behavioural indices. Europ J Pharmacol 1990; 175: 187 195.

47 Vassend O, Skrondal A. The role of negative affectivity in self assessment of health. J Health Psych 1999; 4: $465-$ 482. 